\title{
O USO DE ESTRATÉGIAS DE REPARO NO CONSTITUINTE ONSET COMPLEXO: DESENVOLVIMENTO FONOLÓGICO NORMAL E DESVIANTE
}

\section{The use of repair strategies in the complex onset constituent: normal phonological development and phonological disorder}

\author{
Janaína Sofia Baesso ${ }^{(1)}$, Helena Bolli Mota ${ }^{(2)}$, \\ Carolina Lisbôa Mezzomo ${ }^{(3)}$, Simone Weide Luiz ${ }^{(4)}$
}

\begin{abstract}
RESUMO
Objetivo: descrever e analisar o uso das estratégias de reparo empregadas por crianças com aquisição fonológica normal e desviante, no constituinte silábico onset complexo, a fim de verificar as semelhanças e as diferenças existentes entre os grupos em estudo. Método: foram utilizados dados de fala de 60 crianças com aquisição normal, 30 meninos e 30 meninas, e 84 crianças com aquisição desviante, 53 meninos e 31 meninas. A idade do grupo normal variou de 1:0 a 4:0, enquanto que a do grupo desviante variou de 3:0 a 11:0 anos. Foram consideradas as variáveis: idade, sexo, contexto precedente e seguinte, obstruinte do onset complexo, tipo de líquida do onset complexo, tonicidade, número de sílabas e posição na palavra. Posteriormente, os dados foram submetidos à análise estatística por meio do programa VARBRUL. Resultados: as crianças com desenvolvimento fonológico normal e desviante utilizaram os seguintes recursos, respectivamente: simplificação para $\mathrm{C}^{1} \mathrm{~V}(93 \%$; $77 \%)$, alteração do traço da obstruinte (5\%; 17\%), alteração do traço da líquida (1\%; $5 \%$ ), epêntese $(1 \% ; 0 \%)$ e metátese $(0 \% ; 1 \%)$. As variáveis tonicidade, contexto precedente e posição na palavra não foram relevantes no estudo. Conclusão: o recurso mais frequente foi o de simplificação para $\mathrm{C}^{1} \mathrm{~V}$ em ambos os grupos, contudo, outras estratégias foram aplicadas, principalmente, pelo grupo desviante. Quanto à influência das variáveis, concluiu-se que as variáveis idade, sexo, tipo de obstruinte e tipo de líquida do onset complexo, contexto seguinte e número de sílabas influenciaram de alguma forma os recursos estudados.
\end{abstract}

DESCRITORES: Fala; Desenvolvimento de Linguagem; Linguagem Infantil; Distúrbios da Fala

(1) Fonoaudióloga clínica e do Serviço Social da Indústria SESI, RS; Mestre em Distúrbios da Comunicação Humana pela Universidade Federal de Santa Maria, UFSM, Santa Maria, RS.

(2) Fonoaudióloga; Professora Adjunta do Departamento de Fonoaudiologia da Universidade Federal de Santa Maria, UFSM, Santa Maria, RS; Doutora em Letras pela Pontifícia Universidade Católica do Rio Grande do Sul.

(3) Fonoaudióloga; Professora Adjunta do Departamento de Fonoaudiologia da Universidade Federal de Santa Maria, UFSM, Santa Maria, RS; Doutora em Letras pela Pontifícia Universidade Católica do Rio Grande do Sul.

(4) Licenciada em Letras Português/Inglês e respectvas Literaturas; Mestranda do Programa de Pós-Graduação em Distúrbios da Comunicação Humana, UFSM, Santa Maria, RS, Brasil.

Conflito de interesses: inexistente

\section{INTRODUÇÃO}

Ao longo do desenvolvimento fonológico, a criança mostra-se confusa ao se deparar com o sistema fonológico utilizado no meio em que está inserida e, consequentemente, acaba lançando mão de recursos a fim de simplificar suas produções em um movimento de adaptação do output às suas capacidades.

Os "recursos" utilizados também são denominados estratégias de reparo, e referem-se àquilo que as crianças realizam em lugar do segmento e/ou da estrutura silábica que ainda não conhecem ou cuja produção não dominam ${ }^{1}$. Essas estratégias são observadas tanto durante a aquisição 
fonológica normal quanto desviante, porém com diferença cronológica, ou seja, as estratégias podem perdurar por mais tempo em casos de desvio ${ }^{2}$.

Ainda, as estratégias de reparo aplicadas podem remeter às dificuldades que a criança está enfrentando e, também, podem mostrar o nível de consciência que a criança tem da própria fala ${ }^{3}$. Por isso, as crianças com desvio fonológico evolutivo (DFE) têm, de modo geral, um nível de consciência metalinguística inferior quando comparadas a crianças com desenvolvimento fonológico normal (DFN) ${ }^{4}$.

No que se refere ao tipo de estratégia de reparo a ser aplicada pelas crianças, estudos que descrevem a fonologia do Português Brasileiro (PB) constatam que a posição que os segmentos ocupam na palavra e na sílaba é de grande relevância para o tipo de alteração que os mesmos irão sofrer ${ }^{5-7}$.

Em relação ao constituinte silábico onset complexo, pesquisas constataram que esta é a última estrutura silábica a atingir estabilidade dentro do sistema fonológico ${ }^{8,9}$. Outras pesquisas ${ }^{7,10-13}$ detectaram ser a simplificação do onset complexo a estratégia mais adotada neste constituinte. Contudo, outros recursos também são observados, tais como alteração do traço da obstruinte, metátese, epêntese, entre outros. Quando utilizada a análise acústica, o emprego da estratégia de alongamento compensatório, que é um prolongamento temporal em relação ao alvo-adulto, é evidenciado ${ }^{8}$.

Alguns autores 7,11,13,14 perceberam em seus estudos que existe uma tendência de as crianças com DFE utilizarem os mesmos tipos de estratégias de reparo que as crianças mais novas com DFN.

A aquisição fonológica normal pode ser definida como aquela em que o domínio fonológico ocorre de forma espontânea, dentro de uma determinada faixa etária comum à maior parte das crianças. Assim, há o estabelecimento de um sistema fonológico condizente com o sistema fonológico adulto ${ }^{1,15}$. Contudo, durante o processo de aquisição fonológica ocorrem regressões de uso, tanto na aquisição normal quanto na desviante ${ }^{16}$.

Quando o desempenho fonológico da criança apresenta-se aquém do esperado, pode-se ter um DFE, que é uma desorganização, inadaptação ou anormalidade do sistema fonológico da criança em relação ao sistema-alvo de sua comunidade linguística, na ausência de comprometimentos orgânicos ${ }^{17,18}$. Há uma dificuldade de organização mental e de adequação ao input recebido ${ }^{1,15}$. Embora a causa do DFE não esteja claramente definida, esta atipia é caracterizada por alterações na produção dos sons e uso inadequado das regras fonológicas da língua ${ }^{19}$.
Os desvios fonológicos podem ser diferenciados em termos de gravidade conforme o tipo de estratégia de reparo utilizada, observando-se se o recurso evidencia ou não algum tipo de conhecimento fonológico a respeito do fonema alvo ${ }^{6}$.

Neste contexto, tem-se como objetivo desta pesquisa descrever e analisar o uso das estratégias de reparo empregadas por crianças com DFN e DFE, no constituinte silábico onset complexo, a fim de verificar as semelhanças e as diferenças existentes no uso dos recursos empregados pelas crianças dos diferentes grupos.

\section{MÉTODO}

A presente pesquisa caracterizou-se por ser uma análise de dados de fala, tipo transversal e de cunho quanti-qualitativo.

Foram utilizados dados de fala de 60 crianças com DFN, 30 meninos e 30 meninas, e 84 sujeitos com DFE, 53 meninos e 31 meninas. A idade dos informantes com DFN variou de 1:0 a 4:0, enquanto que a idade das crianças com DFE variou de 3:0 a 11:0 anos.

O critério de seleção para definição da idade mínima das crianças com DFN baseou-se em estudos sobre a aquisição da linguagem, os quais mostram que, com um ano de idade, a criança inicia suas produções. Assim, adotou-se esta idade com o intuito de investigar o uso das estratégias de reparo desde o seu surgimento.

Quanto à idade máxima, pesquisas prévias na área mencionam os 4 anos de idade como um marco do desenvolvimento fonológico, pois, nesse período, fica claro o domínio dos segmentos consonantais que a criança já possui sobre a língua na qual está inserida.

Em relação às crianças com aquisição desviante, a idade mínima estabelecida foi de três anos, pois neste período já é possível constatar que o desenvolvimento fonológico da criança não é condizente com a sua idade, caracterizando um DFE. O limite máximo adotado foi de onze anos, visto que foi utilizado o maior número de sujeitos possível do banco de dados com DFE.

É importante mencionar que as crianças com DFE em idade acima de 9 anos foram avaliadas, a fim de descartar os casos de alteração de fala considerados como 'erros residuais de fala'. De acordo com alguns estudos sobre a aquisição do Inglês Americano ${ }^{(20,21)}$, as crianças que não são capazes de produzir todo o inventário fonético da sua língua até os nove anos de idade apresentam os chamados 'erros residuais de fala'. Essas crianças apresentam falha na articulação de fonemas, dentre os quais se destacam os fonemas 
/s, z, r, l/. Os erros geralmente se referem à distorção de fonemas, mas também podem envolver substituições e omissões.

Todos os sujeitos que compõem a amostra são monolíngues, falantes do português brasileiro.

Os dados de fala do grupo com DFN foram obtidos por meio de um acompanhamento transversal, em entrevistas mensais que variavam de 25 a 45 minutos, dependendo da tolerância de cada criança.

As coletas tinham como objetivo eliciar a fala e a nomeação espontânea das crianças e/ou, eventualmente, a imitação retardada. Para isto, foram utilizados brinquedos, os quais reproduzem a lista de palavras da Avaliação Fonológica da Criança ${ }^{22}$, e também são representativos de uma lista de palavras que opõe as estruturas silábicas CCV X CV e CVC X CV.

As amostras de fala das crianças com DFE também foram obtidas transversalmente, sendo utilizada apenas a avaliação inicial de cada criança. Foi aplicado o instrumento Avaliação Fonológica da Criança ${ }^{22} \mathrm{com}$ as cinco figuras temáticas, para a coleta da fala das crianças.

Além disso, os sujeitos com aquisição desviante foram submetidos a avaliações fonoaudiológicas, incluindo avaliação da linguagem compreensiva e expressiva, do sistema estomatognático, da discriminação auditiva, da voz, da fonologia, e avaliação audiológica. As crianças também foram submetidas às avaliações complementares, envolvendo avaliação otorrinolaringológica e neurológica.

Após as gravações, os registros de fala das crianças com DFN e DFE foram transcritos e revistos por mais dois julgadores separadamente, com experiência em transcrição fonética. Quando não houve consenso sobre as palavras transcritas, essas foram descartadas para maior confiabilidade dos dados.

É importante destacar que os transcritores dos registros de fala de crianças com DFN possuíam experiência em análise acústica, e possivelmente, uma percepção auditiva mais refinada que aqueles que não têm essa experiência.

Os critérios de inclusão para o grupo com DFN foram: apresentar desenvolvimento fonológico normal; idade entre um e quatro anos; podendo ser de ambos os sexos. Sujeitos submetidos a tratamento fonoaudiológico anterior à data da coleta; com alterações audiológicas; e que apresentassem alterações neurológicas, psicológicas e cognitivas evidentes, foram excluídos da pesquisa.

Para as crianças com DFE, os critérios de inclusão foram: apresentar diagnóstico de desvio fonológico evolutivo; idade entre três e onze anos; podendo ser de ambos os sexos. Assim como no DFN, crianças submetidas a tratamento fonoaudiológico anterior à data da coleta; com alterações evidentes nos aspectos neurológico, cognitivo, psicológico e/ou emocional; e com alterações audiológicas foram excluídas do estudo.

Após a seleção das amostras de fala, foi realizado o levantamento das estratégias de reparo empregadas no constituinte silábico coda por crianças com DFN e DFE.

As estratégias de reparo observadas foram denominadas variáveis linguísticas dependentes, e consideraram-se as seguintes possibilidades: simplificação para $C^{\prime} V$, simplificação para $C^{2} V$, simplificação para $V$, omissão da sílaba alvo, alteração do traço da obstruinte, alteração do traço da líquida, alongamento compensatório, metátese, epêntese e outras realizações.

Como fatores intervenientes no uso dos recursos, foram consideradas as variáveis extralinguísticas independentes idade e sexo; e as linguísticas independentes contexto precedente e seguinte, obstruinte do onset complexo, tipo de líquida do onset complexo, tonicidade, número de sílabas e posição na palavra. Foram selecionados estes fatores, pois pesquisas prévias ${ }^{6,10,12,13,15}$, já verificaram a relevância destas variáveis em seus estudos sobre aquisição fonológica.

Especificamente em relação à variável linguística posição na palavra, foram consideradas as diferentes posições de onset complexo: inicial (ex.: prato), medial (ex.: estrada) e final (ex.: cobra).

Em seguida ao levantamento das estratégias de reparo em coda, estas foram codificadas em formulários específicos à cada grupo, um para as crianças com DFN e outro para as crianças com DFE, no Microsoft Access 2003.

Por meio da codificação constatou-se um total de 883 estratégias de reparo para o grupo com DFN e 2529 para o grupo com DFE, em um total de 3412 recursos. Cada recurso foi contabilizado separadamente e equivaleu a uma entrada, ou seja, se na mesma palavra houvesse duas estratégias de reparo, foram consideradas duas entradas, por exemplo: brabo $\rightarrow$ papu, neste caso foi contabilizado o recurso de simplificação para $\mathrm{C}^{1} \mathrm{~V}$ e alteração do traço da obstruinte - duas entradas. Vale ressaltar que todas as produções corretas da criança não foram consideradas.

A pesquisa foi realizada a partir do levantamento de estratégias de reparo em dois bancos de dados. O banco de dados que contém amostras de fala de crianças com DFN foi criado a partir da realização de um projeto, o qual obteve aprovação do Comitê de Ética e Pesquisa de uma Instituição de Ensino Superior, com cadastro sob número 064/2004. No que concerne às crianças com DFE, 
o banco de dados diz respeito a registros de fala obtidos mediante a execução de um segundo projeto, também aprovado pelo Comitê de Ética e Pesquisa de uma Instituição de Ensino Superior, com cadastro sob número 6331.

Posteriormente à codificação, os dados foram submetidos à análise estatística por meio do Pacote Computacional VARBRUL ${ }^{23}$. Esse conjunto de programas é largamente utilizado em análises linguísticas variacionistas ${ }^{24}$. Entretanto, apesar de ser um programa específico para a área da variação linguística, diversos estudos anteriores ${ }^{5,7,10-12,13}$ comprovaram a eficiência do programa para analisar dados linguísticos em grande quantidade, fornecendo frequências e probabilidades, além de selecionar variáveis relevantes no processo de aquisição da linguagem. O programa faz a análise probabilística na forma binária, atribuindo pesos relativos às variantes das variáveis independentes, com relação à variável dependente. Além disso, o VARBRUL trabalha com uma margem de erro de $5 \%$, mostrando que qualquer fator com significância abaixo desse valor não era estatisticamente expressivo. Os valores probabilísticos são retirados da interação que contém, conjuntamente, todas as variáveis selecionadas pelo programa. Esses fatores são estatisticamente significantes e mostram que exercem um papel no fenômeno estudado. Assim, valores probabilísticos entre .50 e .59 foram considerados neutros; enquanto os valores iguais ou superiores a .60 foram favorecedores e, por último, valores inferiores a .50 foram desfavorecedores.

\section{RESULTADOS}

A partir da análise dos dados, observou-se que o número de estratégias de reparo utilizadas pelos grupos com DFN e DFE foi o mesmo, ambos os grupos utilizaram quatro estratégias distintas.

A partir da Figura 1, pôde-se perceber que as estratégias simplificação para $\mathrm{C}^{1} \mathrm{~V}$ (ex.: preto $\rightarrow$ ['petu]), alteração do traço da obstruinte (ex.: brabo $\rightarrow$ ['prabu]) e alteração do traço da líquida (ex.: prato $\rightarrow$ ['platu]) foram empregadas por ambos os grupos. A diferença residiu no fato de as crianças com DFN lançarem mão da estratégia de epêntese (ex.:livro $\rightarrow$ ['livuru]) enquanto os sujeitos com DFE empregaram a metátese (ex.: primo $\rightarrow$ ['pirmu]).

Estratégias de reparo usadas por crianças com aquisição fonológica normal e desviante na posição de onset complexo

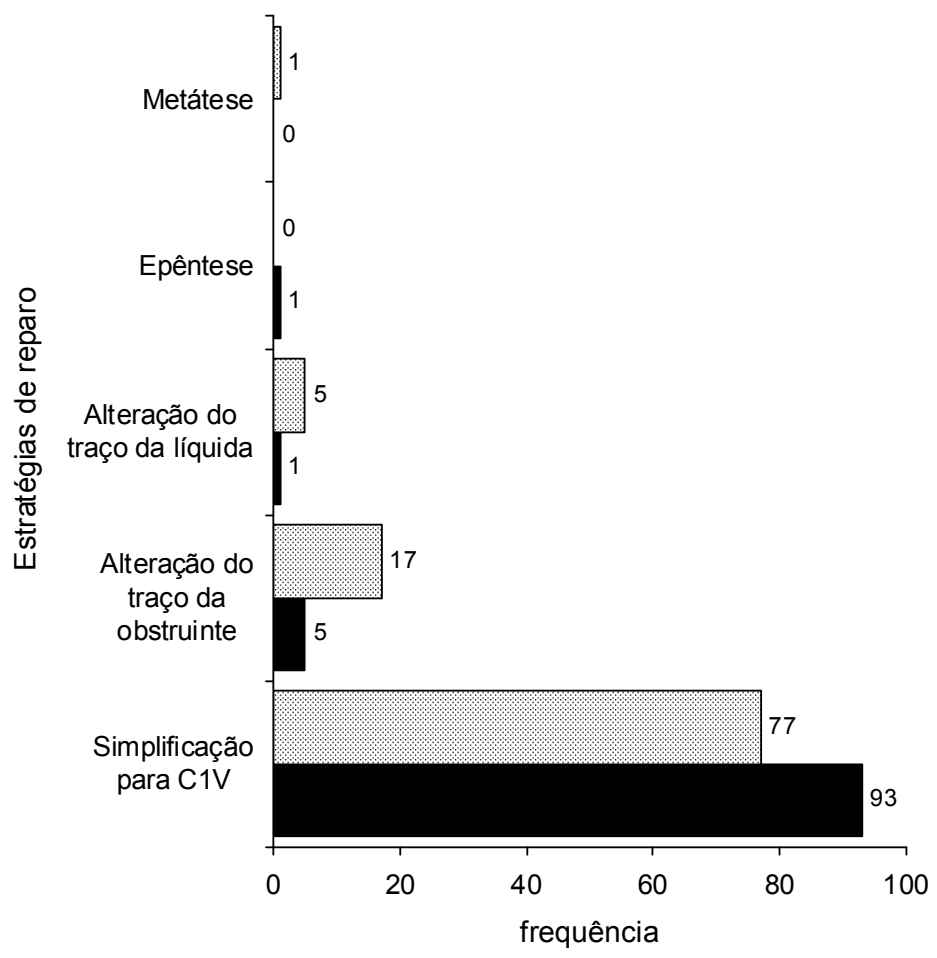

Aquisição típica Aquisição atípica

Figura 1 - Frequência e tipo de estratégias de reparo utilizadas por crianças com DFN e DFE no OC 
A estratégia simplificação para $C^{1} \mathrm{~V}$ mostrou-se o recurso mais numeroso em ambos os grupos, com uma frequência de $93 \%$ para crianças com DFN e de $77 \%$ para as crianças com desvio fonológico.

Observou-se o recurso alteração do traço da obstruinte em $5 \%$ das crianças com DFN e $17 \%$ das crianças com DFE, sendo o segundo recurso mais utilizado somando-se as realizações de ambos os grupos.

A estratégia de reparo alteração do traço de líquida constituiu uma frequência baixa, de 1\% para o grupo com DFN e $5 \%$ para o grupo com DFE. Quanto aos recursos de estrutura silábica, a metátese (DFE) e a epêntese (DFN) ocorreram em apenas $1 \%$ das amostras.

Em função da frequência de ocorrência de cada estratégia de reparo, os recursos que permitiram a realização da análise estatística foram: simplificação para $C^{1} \mathrm{~V}$ e alteração do traço da obstruinte (DFN e DFE), e alteração do traço da líquida e metátese (DFE).

A seguir serão expostas as Tabelas com as variáveis selecionadas como relevantes para o uso das estratégias de reparo em estudo, bem como o papel das variantes.

\section{Simplificação para $\mathbf{C}^{1} \mathbf{V}$}

As variáveis comuns selecionadas como significantes no uso da simplificação para $\mathrm{C}^{1} \mathrm{~V}$ (ex.: brabo $\rightarrow$ ['babu]) para os grupos DFN e DFE foram obstruinte do onset complexo $(O C)$ e idade. No que se refere à primeira, a obstruinte plosiva surda (ex.: preto $\rightarrow$ ['petu]) foi a única variante que favoreceu o uso do recurso para as crianças com DFN (Tabela 1) e com DFE (Tabela 2).

Tabela 1 - Variáveis selecionadas como estatisticamente significantes no emprego da simplificação do OC para o grupo com DFN

\begin{tabular}{lrccc}
\hline Simplificação para $\mathbf{C}^{1} \mathbf{V}$ & & Frequência & $\%$ & $\begin{array}{c}\text { Peso } \\
\text { Relativo }\end{array}$ \\
\hline Obstruinte do OC & Plosiva coronal sonora & $0 / 36$ & 0 & .00 \\
& Fricativa labial sonora & $0 / 50$ & 0 & .00 \\
& Fricativa labial surda & $30 / 36$ & 83 & .15 \\
& Plosiva dorsal sonora & $61 / 79$ & 77 & .21 \\
& Plosiva dorsal surda & $12 / 13$ & 92 & .22 \\
& Plosiva labial sonora & $205 / 230$ & 89 & .35 \\
& Plosiva coronal surda & $273 / 290$ & 94 & .56 \\
& Plosiva labial surda & $88 / 89$ & 99 & .94 \\
\hline Idade & $3: 6 ; 0-3: 8 ; 29$ & $0 / 43$ & 0 & .00 \\
& $3: 9 ; 0-3: 11 ; 29$ & $0 / 26$ & 0 & .00 \\
& $1: 6 ; 0-1: 8 ; 29$ & $69 / 83$ & 83 & .12 \\
& $1: 9 ; 0-1: 11 ; 29$ & $41 / 50$ & 82 & .17 \\
& $2: 9 ; 0-2: 11 ; 29$ & $87 / 98$ & 89 & .34 \\
& $3: 0 ; 0-3: 2 ; 29$ & $66 / 79$ & 84 & .35 \\
& $2: 3 ; 0-2: 5 ; 29$ & $100 / 110$ & 91 & .46 \\
& $2: 0 ; 0-2: 2 ; 29$ & $113 / 120$ & 94 & .82 \\
& $2: 6 ; 0-2: 8 ; 29$ & $109 / 111$ & 98 & .82 \\
& $3: 3 ; 0-3: 5 ; 29$ & $84 / 86$ & 98 & .84 \\
\hline Contexto seguinte & Vogal dorsal & $205 / 238$ & 86 & .31 \\
& Vogal labial/dorsal & $286 / 308$ & 93 & .55 \\
\hline
\end{tabular}

Em relação à variável idade, pode-se perceber que a estratégia foi utilizada ao longo do percurso de aquisição de forma aleatória, ou seja, algumas faixas etárias iniciais favoreceram o uso do recurso, ao mesmo tempo em que crianças com idade mais avançada também empregaram o recurso de forma significante em ambos os grupos.
Os grupos também apresentaram variáveis singulares, tais como o contexto seguinte para as crianças com DFN (Tabela 1), e número de sílabas e líquida do onset complexo para as crianças com DFE (Tabela 2). 
Tabela 2 - Variáveis selecionadas como estatisticamente significantes no emprego da simplificação do OC para o grupo com DFE

\begin{tabular}{lrccc}
\hline Simplificação para $C^{1} \mathbf{V}$ & & Frequência & $\%$ & $\begin{array}{c}\text { Peso } \\
\text { Relativo }\end{array}$ \\
\hline Obstruinte do OC & Plosiva coronal sonora & $0 / 36$ & 0 & .00 \\
& Fricativa labial sonora & $0 / 50$ & 0 & .00 \\
Fricativa labial surda & $30 / 36$ & 83 & .15 \\
& Plosiva dorsal sonora & $61 / 79$ & 77 & .21 \\
& Plosiva dorsal surda & $12 / 13$ & 92 & .22 \\
& Plosiva labial sonora & $205 / 230$ & 89 & .35 \\
Plosiva coronal surda & $273 / 290$ & 94 & .56 \\
& Plosiva labial surda & $88 / 89$ & 99 & .94 \\
\hline Idade & $3: 6 ; 0-3: 8 ; 29$ & $0 / 43$ & 0 & .00 \\
& $3: 9 ; 0-3: 11 ; 29$ & $0 / 26$ & 0 & .00 \\
& $1: 6 ; 0-1: 8 ; 29$ & $69 / 83$ & 83 & .12 \\
& $1: 9 ; 0-1: 11 ; 29$ & $41 / 50$ & 82 & .17 \\
& $2: 9 ; 0-2: 11 ; 29$ & $87 / 98$ & 89 & .34 \\
& $3: 0 ; 0-3: 2 ; 29$ & $66 / 79$ & 84 & .35 \\
& $2: 3 ; 0-2: 5 ; 29$ & $100 / 110$ & 91 & .46 \\
& $2: 0 ; 0-2: 2 ; 29$ & $113 / 120$ & 94 & .82 \\
& $2: 6 ; 0-2: 8 ; 29$ & $109 / 111$ & 98 & .82 \\
& $3: 3 ; 0-3: 5 ; 29$ & $84 / 86$ & 98 & .84 \\
\hline Contexto seguinte & Vogal dorsal & $205 / 238$ & 86 & .31 \\
& Vogal labial/dorsal & $286 / 308$ & 93 & .55 \\
\hline valor de $\mathrm{p}=0.05$ & Vogal coronal & $178 / 191$ & 93 & .66 \\
\hline
\end{tabular}

No que concerne ao grupo com desenvolvimento fonológico normal, o contexto seguinte vogal coronal (ex.: primo $\rightarrow$ ['pimu]) favoreceu o uso da estratégia de reparo (Tabela 1). Em relação às variantes apontadas como significantes para o grupo com DFE, pôde-se concluir que todas as possibilidades apresentaram um papel neutro e/ou desfavorecedor ao uso da estratégia. As palavras monossílabas (ex.: flor $\rightarrow$ ['for]) e dissílabas (ex.: prato $\rightarrow$ ['patu]), e onset complexo formado por líquida lateral (ex.: pluto $\rightarrow$ ['puto]) foram desfavoráveis ao uso do recurso, enquanto que palavras trissílabas (ex.: brinquedo $\rightarrow$ ['bin'kedu]) e polissílabas (ex.: bicicleta $\rightarrow$ [bisi'klkta]) e onset complexo formado por líquida não lateral (ex.: livro $\rightarrow$ ['livu]) atuaram de forma neutra (Tabela 2).

\section{Alteração do traço da obstruinte}

Os resultados estatísticos relacionados à estratégia de alteração do traço da obstruinte do $O C$ (ex.: branco $\rightarrow$ ['pãnku], livro $\rightarrow$ ['lifru]) apontaram as variáveis tipo de obstruinte do $\mathrm{OC}$, idade e sexo como estatisticamente significantes para ambos os grupos. Quanto à variável idade, as crianças com DFN com idades de 1:6;0 - 1:8;29 e 1:9;0 1:11;29 (tabela 3), apresentaram maior probabilidade de lançar mão da estratégia alteração do traço da obstruinte, e para os sujeitos com DFE, as faixas etárias medianas atuaram de forma neutra, enquanto que as faixas etárias limítrofes, mais precoces de 3 a 5 anos e mais tardias de 9 a 11 anos (tabela 4), desfavoreceram o uso da estratégia em questão. 
Tabela 3 - Variáveis selecionadas como estatisticamente significantes no emprego de recursos que envolvem alteração valor traço no OC - grupo DFN

\begin{tabular}{lrccc}
\hline Alteração do traço da obstruinte & & Frequência & $\%$ & $\begin{array}{c}\text { Peso } \\
\text { Relativo }\end{array}$ \\
\hline Idade & $3: 6 ; 0-3: 8 ; 29$ & $0 / 43$ & 0 & .00 \\
& $3: 9 ; 0-3: 11 ; 29$ & $0 / 26$ & 0 & .00 \\
& $3: 3 ; 0-3: 5 ; 29$ & $2 / 73$ & 3 & .19 \\
& $2: 9 ; 0-2: 11 ; 29$ & $1 / 71$ & 1 & .19 \\
& $2: 6 ; 0-2: 8 ; 29$ & $2 / 88$ & 2 & .33 \\
& $2: 3 ; 0-2: 5 ; 29$ & $4 / 97$ & 4 & .40 \\
& $2: 0 ; 0-2: 2 ; 29$ & $6 / 108$ & 6 & .49 \\
& $3: 0 ; 0-3: 2 ; 29$ & $13 / 63$ & 21 & .66 \\
\hline Obstruinte do OC & $1: 9 ; 0-1: 11 ; 29$ & $4 / 34$ & 12 & .76 \\
& $1: 6 ; 0-1: 8 ; 29$ & $14 / 72$ & 19 & .96 \\
\hline Sexo & Fricativa labial sonora & $0 / 36$ & 0 & .00 \\
& Plosiva labial surda & $0 / 50$ & 0 & .00 \\
& Plosiva coronal sonora & $0 / 28$ & 0 & .00 \\
& Plosiva coronal surda & $11 / 259$ & 4 & .29 \\
& Plosiva labial sonora & $16 / 230$ & 7 & .55 \\
& Fricativa labial surda & $1 / 25$ & 4 & .68 \\
Contexto seguinte & Plosiva dorsal sonora & $17 / 79$ & 22 & .84 \\
& Plosiva dorsal surda & $1 / 13$ & 8 & .93 \\
\hline * valor de $\mathrm{p}=.015$ & Feminino & $12 / 275$ & 4 & .31 \\
\hline & Masculino & $34 / 331$ & 10 & .66 \\
\hline
\end{tabular}

Em relação à variável tipo de obstruinte do OC, para as crianças com DFN as plosivas dorsais surda (ex.: creme $\rightarrow$ ['temi]) e sonora (ex.: grilo $\rightarrow$ ['krilu]) e a fricativa labial surda (ex.: fraco $\rightarrow$ ['saku]) (tabela 3) atuaram de forma favorável ao uso do recurso. Para o grupo com DFE, os contextos plosiva dorsal sonora (ex.: grama $\rightarrow$ ['kãma]), fricativa labial sonora (ex.: livro $\rightarrow$ ['lifu]) e plosiva coronal sonora (ex.: dragão $\rightarrow$ [tra'gãw]) (tabela 4) cumpriram papel favorável ao emprego da estratégia.

A variável sexo revelou que para as crianças com DFN o sexo masculino atuou de forma favorável, enquanto que o sexo feminino desfavoreceu o uso da estratégia (Tabela 3). Para o grupo com DFE, os meninos apresentaram probabilidade neutra no emprego da estratégia, enquanto que as meninas apresentaram papel desfavorecedor (Tabela 4).

Para o grupo com DFN, ainda foi selecionada a variável contexto seguinte, sendo a vogal dorsal (ex.: brabo $\rightarrow$ ['pabu]) favorecedora e vogal coronal (ex.: primo $\rightarrow$ ['timu]) desfavorecedora ao emprego deste recurso (Tabela 3 ).
Para as crianças com DFE, foi selecionada como significante a variável número de sílabas, sendo seu papel neutro (palavras mono, di e polissilábicas; flor $\rightarrow$ ['por], livro $\rightarrow$ ['lifru], bicicleta à [bisi'tcta]) ou desfavorecedor (palavras trissílabas (ex.: brinquedo à [prinkedu) à aplicação da estratégia (Tabela 4).

\section{Alteração do traço da líquida}

As variáveis selecionadas pelo programa estatístico como significantes ao uso da estratégia de alteração do traço da líquida (ex.: branco $\rightarrow$ [blãjku]) para o grupo com DFE foram sexo, idade, tipo de obstruinte do OC e número de sílabas (Tabela 4).

Assim, os resultados revelaram que crianças de sexo masculino, obstruintes do OC preenchidas por plosiva labial sonora e surda (ex.: brabo $\rightarrow$ ['blabu], praça $\rightarrow$ ['plasa]), e plosiva dorsal surda (ex.: cravo $\rightarrow$ ['klavu]), e palavras monossílabas (ex.: três à ['tles]) favoreceram a aplicação da estratégia em questão (Tabela 4).

Em relação à variável idade, constatou-se que as faixas etárias medianas atuaram de forma 
Tabela 4 - Variáveis selecionadas como estatisticamente significantes no emprego de recursos que envolvem alteração do valor do traço ou estrutura silábica no OC - grupo DFE

\begin{tabular}{|c|c|c|c|c|}
\hline \multicolumn{2}{|c|}{ Alteração do traço da obstruinte } & \multirow{2}{*}{$\begin{array}{c}\text { Frequência } \\
\text { 9/281 }\end{array}$} & \multirow{2}{*}{$\begin{array}{l}\% \\
3\end{array}$} & \multirow{2}{*}{$\begin{array}{c}\begin{array}{c}\text { Peso } \\
\text { relativo }\end{array} \\
.14\end{array}$} \\
\hline Obstruinte do OC & Plosiva labial surda & & & \\
\hline & Plosiva coronal surda & $46 / 503$ & 9 & .37 \\
\hline & Plosiva labial sonora & $92 / 532$ & 17 & .55 \\
\hline & Fricativa labial surda & $46 / 284$ & 16 & .55 \\
\hline & Plosiva dorsal surda & $43 / 232$ & 19 & .56 \\
\hline & Plosiva coronal sonora & $58 / 284$ & 23 & .63 \\
\hline & Fricativa labial sonora & $54 / 192$ & 28 & .69 \\
\hline & Plosiva dorsal sonora & $75 / 257$ & 29 & .73 \\
\hline \multirow[t]{4}{*}{ Idade } & $3-5$ anos & $60 / 529$ & 11 & .35 \\
\hline & $9-11$ anos & $1 / 19$ & 5 & .36 \\
\hline & $7-9$ anos & $70 / 457$ & 15 & .53 \\
\hline & $5-7$ anos & $292 / 1524$ & 19 & .54 \\
\hline \multirow[t]{2}{*}{ Sexo } & Feminino & $242 / 1532$ & 16 & .47 \\
\hline & Masculino & $181 / 997$ & 18 & .55 \\
\hline \multirow[t]{4}{*}{ Número de sílabas } & Trissilábica & $53 / 466$ & 11 & .40 \\
\hline & Monossilábica & $44 / 302$ & 15 & .52 \\
\hline & Polissilábica & $35 / 205$ & 17 & .52 \\
\hline & Dissilábica & $291 / 1556$ & 19 & .52 \\
\hline \multicolumn{5}{|l|}{${ }^{*}$ valor de $p=.034$} \\
\hline \multicolumn{5}{|c|}{ Alteração do traço da líquida } \\
\hline \multirow[t]{2}{*}{ Sexo } & Feminino & $23 / 298$ & 2 & .36 \\
\hline & Masculino & $94 / 1411$ & 7 & .60 \\
\hline \multirow[t]{4}{*}{ Idade } & $3-5$ anos & $5 / 454$ & 1 & .20 \\
\hline & $9-11$ anos & $1 / 19$ & 5 & .47 \\
\hline & $7-9$ anos & $14 / 457$ & 3 & .55 \\
\hline & $5-7$ anos & $97 / 1409$ & 7 & .59 \\
\hline \multirow[t]{8}{*}{ Obstruinte do OC } & Plosiva coronal sonora & $3 / 233$ & 1 & .22 \\
\hline & Fricativa labial sonora & $3 / 185$ & 2 & .25 \\
\hline & Fricativa labial surda & $12 / 273$ & 4 & .40 \\
\hline & Plosiva dorsal sonora & $9 / 234$ & 4 & .47 \\
\hline & Plosiva coronal surda & $28 / 463$ & 6 & .56 \\
\hline & Plosiva dorsal surda & $10 / 204$ & 5 & .63 \\
\hline & Plosiva labial surda & $16 / 249$ & 6 & .63 \\
\hline & Plosiva labial sonora & $36 / 498$ & 7 & .64 \\
\hline \multirow[t]{4}{*}{ Número de sílabas } & Polissilábica & $4 / 188$ & 2 & .25 \\
\hline & Trissilábica & $22 / 437$ & 5 & .46 \\
\hline & Dissilábica & $69 / 1431$ & 5 & .52 \\
\hline & Monossilábica & $22 / 283$ & 8 & .64 \\
\hline \multicolumn{5}{|l|}{${ }^{*}$ valor de $p=.015$} \\
\hline \multicolumn{5}{|l|}{ Metátese } \\
\hline \multirow[t]{8}{*}{ Obstruinte do OC } & Fricativa labial surda & $0 / 284$ & 0 & .00 \\
\hline & Plosiva dorsal surda & $1 / 193$ & 1 & .19 \\
\hline & Fricativa labial surda & $1 / 252$ & 0 & .28 \\
\hline & Fricativa labial surda & $2 / 438$ & 0 & .36 \\
\hline & Plosiva labial surda & $2 / 281$ & 1 & .39 \\
\hline & Plosiva coronal surda & $4 / 352$ & 1 & .68 \\
\hline & Fricativa labial sonora & $4 / 191$ & 2 & .71 \\
\hline & Plosiva coronal sonora & $9 / 245$ & 4 & .86 \\
\hline${ }^{*}$ valor de $p=.048$ & & & & \\
\hline
\end{tabular}


neutra, enquanto que as faixas etárias limítrofes, de 3 a 5 anos e 9 a 11 anos, desfavoreceram o uso do recurso (Tabela 4 ).

\section{Metátese}

A única variável selecionada como estatisticamente significante na análise da estratégia de metátese (ex.: febre $\rightarrow$ ['feber]) foi o tipo de obstruinte do OC, sendo a plosiva coronal sonora (ex.: dragão $\rightarrow$ [da'grãw]) e surda (ex.: letra $\rightarrow$ ['letar]) e a fricativa labial sonora (ex.: livre $\rightarrow$ ['liver]) as obstruintes favorecedoras ao uso do recurso (Tabela 4).

\section{DISCUSSÃO}

\section{Tipo e frequência das estratégias de reparo empregadas no onset complexo}

Vários autores têm observado que a estratégia de reparo mais frequente durante o percurso de aquisição do onset complexo, quer seja por crianças com DFN ${ }^{8,25}$, quer seja por crianças desviantes ${ }^{7,9,26,27}$, é a simplificação para $\mathbf{C}^{1} \mathbf{V}$. Um estudo detectou que as omissões são mais frequentes do que as substituições em onset complexo ${ }^{28}$. Há também outro estudo no qual a redução do encontro consonantal (ou simplificação para $\mathrm{C}^{1} \mathrm{~V}$ ) é o segundo processo fonológico mais frequente em crianças com DFN, aparecendo logo após a substituição de líquida ${ }^{29}$.

Os resultados da presente pesquisa vão ao encontro dos estudos supracitados, visto que a estratégia de simplificação para $C^{1} \mathrm{~V}$ constituiu uma frequência de $93 \%$ para o grupo com DFN e $77 \%$ para as crianças com DFE.

Um estudo específico sobre o onset complexo ${ }^{9}$ comparou seus resultados aos obtidos em seu trabalho anterior, realizado com crianças com DFN ${ }^{8}$, e constatou que os sujeitos com DFE empregam a estratégia de simplificação para $\mathrm{C}^{1} \mathrm{~V}$ mais frequentemente que os sujeitos com DFN. Este achado não foi observado neste estudo, no entanto, a divergência entre os resultados pode ser justificada pelo fato de que a primeira pesquisa ${ }^{8}$ estudou 0 processo de aquisição do onset complexo até o seu completo domínio, incluindo crianças de até 5:3 anos de idade. Desta forma, as crianças com DFN pesquisadas no estudo supracitado ${ }^{8}$ diminuíram o uso da estratégia simplificação para $\mathrm{C}^{1} \mathrm{~V}$ à medida que a aquisição da estrutura complexa se completava, fato que não ocorreu no presente estudo, visto que a faixa etária máxima considerada foi de 3:11, idade em que o onset complexo ainda encontra-se instável, e o recurso em questão ainda é empregado de forma produtiva.

Além disso, as evidências do input e as capacidades cognitivas e articulatórias vão se desenvolvendo ao longo do percurso fonológico, e com isso, a criança vai especificando os traços que não estavam presentes na sua representação inicial, isto é, os traços marcados ${ }^{30}$. Considerando esta afirmação, pode-se hipotetizar que a criança com DFN, por ser mais jovem, emprega preferencialmente um recurso mais simples e primário ao se deparar com a estrutura complexa CCV, enquanto que a criança com DFE, por ter uma idade mais avançada, possui maiores evidências de input e melhores capacidades cognitivas e articulatórias, as quais permitem que a criança lance mão de recursos mais elaborados, como alteração do traço da líquida e metátese.

A estratégia de reparo alteração do traço da obstruinte foi empregada mais frequentemente pelo grupo desviante, constituindo uma frequência de $17 \%$ para este grupo, e de $5 \%$ para as crianças com DFN.

Os dados de normalidade em relação à estratégia alteração do traço da obstruinte foram semeIhantes em um estudo sobre a estrutura complexa CCV ${ }^{8}$, que obteve resultados um pouco inferiores aos da presente pesquisa, sendo a estratégia de alteração do traço da obstruinte representativa de apenas $1 \%$ do corpus de crianças com DFN.

Em contrapartida, em relação ao desenvolvimento desviante, outro estudo ${ }^{9}$ constatou que as crianças estudadas não empregaram o recurso alteração do traço da obstruinte. Este fato pode ser justificado pela diferença entre o corpus estudado, visto que o estudo ${ }^{9}$ foi realizado com um pequeno número de sujeitos.

O recurso de alteração do traço da líquida foi utilizado pelas crianças com desenvolvimento normal e também desviante, equivalendo a uma frequência de $1 \%$ e $5 \%$, respectivamente.

Estudos específicos sobre a aquisição do onset complexo ${ }^{8,9}$ constataram que o recurso alteração do traço da líquida foi utilizado por $5 \%$ do corpus de crianças com DFN e $9 \%$ do corpus com DFE.

O presente estudo é corroborado pelas pesquisas supracitadas no sentido de que as crianças com DFE empregaram a estratégia alteração do traço da líquida de modo mais produtivo que os sujeitos com DFN.

A partir desde achado, pode-se presumir que as crianças com desenvolvimento desviante, por terem idade mais avançada, possuem maiores evidências de input e capacidades cognitivas e articulatórias mais desenvolvidas ${ }^{30,31}$, o que as permite realizar tentativas de produção do segmento alvo, por meio de substituições da $\mathrm{C}^{2}$, ao invés de simples omissões.

A afirmação acima também pode justificar o fato de somente crianças com DFE lançarem mão do 
recurso de metátese ao se depararem com a estrutura complexa CCV.

Os achados do presente estudo concordam parcialmente com uma pesquisa anterior ${ }^{9}$, que também observou uma baixíssima produtividade da estratégia de metátese, correspondendo a 0,3\% dos recursos empregados pelas crianças com desvio. No entanto, outro trabalho ${ }^{8}$ verificou que as crianças com DFN também lançam mão da estratégia de metátese, de forma ainda mais produtiva que os sujeitos desviantes, com uma frequência de $1,5 \%$, dado este que não foi constatado na presente pesquisa.

A análise das estratégias de reparo que as crianças com DFN e DFE lançaram mão na posição de onset complexo permitiu verificar semelhanças e diferenças quanto ao tipo e a frequência dos recursos que foram utilizados pelos grupos, bem como quanto às variáveis que favoreceram ou desfavoreceram a aplicação destas estratégias.

A seguir, serão tecidos comentários a respeito do papel destas variáveis linguísticas e extralinguísticas no uso das estratégias de reparo no constituinte onset complexo. As variáveis tonicidade, posição na palavra e contexto precedente não foram selecionadas como relevantes para nenhuma estratégia utilizada. Contudo, em um estudo sobre os efeitos da posição na palavra e da tonicidade em palavras contendo o onset complexo, a precisão dos encontros consonantais foi maior em sílabas iniciais e tônicas ${ }^{32}$.

É válido ressaltar que os comentários tecidos em relação às estratégias de reparo pouco frequentes não devem ser vistos como generalizações para o uso de recursos no constituinte onsetcomplexo, pois se referem a menos de $20 \%$ dos dados analisados.

\section{Estratégias de reparo versus variáveis linguísticas e extralinguísticas}

Variável extralinguística idade

A variável extralinguística idade foi bastante importante para o uso das estratégias de reparo no constituinte onset complexo, sendo selecionada como relevante para as estratégias de simplificação para $\mathrm{C}^{1} \mathrm{~V}$, alteração do traço da obstruinte e alteração do traço da líquida.

Em relação à estratégia de reparo mais produtiva, a simplificação para $\mathrm{C}^{1} \mathrm{~V}$, houve maior probabilidade de crianças com DFN em faixas etárias mais iniciais $(2: 0-2: 2)$, e também em faixas etárias mais avançadas (3:3-3:5) empregarem o recurso. Estes resultados concordam com uma pesquisa anterior ${ }^{8}$, que constatou o emprego da estratégia de simplificação para $\mathrm{C}^{1} \mathrm{~V}$ ao longo do percurso de aquisição, não havendo diferenças quanto à frequência do recurso em diferentes faixas etárias.
De modo contrário, outro estudo ${ }^{10}$ constatou que crianças mais jovens aplicam a estratégia de simplificação para $\mathrm{C}^{1} \mathrm{~V}$ mais frequentemente que crianças em idade mais avançada.

Em relação às estratégias de reparo utilizadas de forma pouco frequente, como as que envolvem o traço da obstruinte, um estudo sobre a aquisição do onset complexo por crianças com DFN mencionou que estes recursos foram mais utilizados por crianças em faixas etárias iniciais ${ }^{8}$.

No presente estudo, o recurso alteração do traço da obstruinte foi empregado de forma significante por crianças em faixas etárias iniciais (1:6 a 1:11), porém, embora com menor probabilidade, a estratégia também foi utilizada por sujeitos com idade mais avançada (3:0 a 3:2). Pode-se pensar que o predomínio do recurso em fases iniciais ocorreu pelo fato de crianças mais jovens empregarem diversas estratégias de reparo em todas as posições silábicas, pois seu sistema fonológico encontra-se ainda muito imaturo e instável.

No que concerne às crianças com aquisição desviante, o recurso simplificação para $C^{1} V$ foi aplicado de forma significante pelas crianças que pertenciam às faixas etárias limítrofes, isto é, $3-5$ anos e 9-11 anos, enquanto que, para os recursos alteração do traço da obstruinte e da líquida, as crianças das faixas etárias intermediárias foram as que mais utilizaram os recursos. Por se tratarem de crianças com DFE, embora não se tenha averiguado diretamente a influência do grau de severidade do desvio fonológico, pode-se considerar a possibilidade de a gravidade do desvio fonológico estar interferindo de forma mais significante do que a faixa etária, o que justificaria o uso "aleatório" do recurso.

\section{Variável extralinguística sexo}

A variável sexo foi importante para os recursos alteração do traço da obstruinte e alteração do traço da líquida, sendo as crianças do sexo masculino as que apresentaram maior probabilidade de aplicar as estratégias.

Os resultados obtidos diferem dos achados encontrados em pesquisas prévias. Ao observar a relação da variável sexo e da ocorrência de estratégias de reparo, uma pesquisa sobre a aquisição das líquidas não laterais ${ }^{9} \mathrm{e}$ outros estudos a respeito do onset complexo ${ }^{10,33}$, constataram que não houve diferenças entre o sexo feminino e masculino. Corroborando estes trabalhos, uma pesquisa com crianças de classe socioeconômica alta ${ }^{34}$ constatou que não houve diferença entre os erros de fala considerando a variável sexo.

Em contrapartida, um trabalho sobre a aquisição da coda ${ }^{6}$ e outro a respeito do desenvolvimento 
fonológico de pré-escolares ${ }^{29}$ verificaram um maior número de estratégias de reparo para o sexo feminino, enquanto os meninos apresentaram maior número de produções corretas. Complementando o que foi citado, uma pesquisa sobre tarefas de nomeação rápida observou que as crianças do sexo feminino apresentaram em média mais erros durante a realização de uma prova ${ }^{35}$.

De modo contrário, uma pesquisa ${ }^{5}$ observou resultados semelhantes aos do presente estudo, e concluiu que as meninas produziram o constituinte onset complexo mais corretamente que os meninos.

Vários autores têm verificado uma relação significante entre desvio fonológico e sujeitos do sexo masculino 1,13,17. Estes trabalhos sustentam os achados obtidos nesta pesquisa, visto que houve maior probabilidade de os meninos lançarem mão das estratégias de reparo estudadas.

Dessa forma, observa-se que existe divergência de resultados na literatura. Assim, acredita-se na necessidade da realização de novos estudos que relacionem o uso de estratégias de reparo à variável sexo, a fim de investigar mais profundamente o papel dessa variável especificamente em relação aos recursos que as crianças lançam mão no percurso de aquisição.

Variável linguística obstruinte do onset complexo

A primeira consoante do onset complexo, a obstruinte, pode exercer influência na produção correta da estrutura CCV, considerando o ponto e o modo de articulação da obstruinte ${ }^{8}$. A partir disso, pode-se refletir se estes contextos favorecedores à produção correta não poderiam também atuar de forma relevante quanto ao tipo de estratégia de reparo a ser selecionada pela criança.

Os resultados do presente trabalho, no que concerne à relevância desta variável para o emprego da estratégia simplificação para $C^{1} \mathrm{~V}$, apontaram para a obstruinte plosiva labial surda como favorecedora ao uso do recurso, tanto para o grupo com DFN, como para o grupo com DFE. Estes achados não concordam com um estudo anterior sobre a aquisição do onset complexo ${ }^{8}$, que verificou que onsets complexos formados por plosivas labiais surda e sonora favorecem as produções corretas do constituinte silábico.

De forma semelhante ao estudo supracitado, outra pesquisa ${ }^{36}$ verificou uma facilidade do traço labial para o domínio do onset complexo, descrevendo a seguinte ordem de aquisição: grupos com obstruinte labial, seguidos por grupos com coronal e por último com dorsal.

Para a estratégia alteração do traço da obstruinte, houve maior probabilidade de ocorrência deste recurso em onsets formados por plosivas dorsais surdas e sonoras, e fricativa labial surda, no grupo com DFN. Os resultados obtidos para o grupo com DFE foram distintos, sendo os onsets complexos formados por fricativa labial sonora, plosiva dorsal sonora e plosiva coronal sonora os que apresentaram maiores pesos relativos, sendo favorecedores ao uso do recurso.

Embora os grupos tenham apresentado resultados diferentes, verificou-se uma ordem de complexidade, ou seja, obstruintes com traços mais complexos são mais suscetíveis a sofrerem a estratégia de alteração do traço da obstruinte, fato não observado na estratégia de simplificação para $\mathrm{C}^{1} \mathrm{~V}$, que a dificuldade pareceu estar relacionada à realização da sílaba complexa e não à obstruinte que compunha o onset complexo.

Desta forma, pode-se pensar que em relação às crianças com DFN, o traço [dorsal] da obstruinte foi o mais complexo para a criança realizar, seguido do traço [contínuo]. Considerando o desenvolvimento fonológico normal, este resultado é sustentado por estudos anteriores que referem uma aquisição mais tardia dos segmentos plosivos dorsais na posição de onset ${ }^{1}$.

A respeito das dificuldades observadas pelo grupo desviante, verificou-se que as dificuldades quanto às obstruintes foram mais variadas, fato que pode ser justificado por se tratar de um grupo com aquisição desviante. Contudo, pode-se hipotetizar que as crianças tenham apresentado dificuldades para realizar traços mais complexos, como [contínuo], [dorsal], e principalmente o [sonoro], neste caso, utilizando o traço não marcado [- sonoro].

No que concerne ao uso da estratégia alteração do traço da líquida, novamente as obstruintes plosivas labiais surda e sonora, acrescidas da plosiva dorsal surda, constituíram contextos favorecedores. Estes resultados não concordam com os obtidos em outro estudo ${ }^{8}$, que constatou que onsets complexos formados por obstruintes com modo de consoante plosivo favorecem a produção correta do constituinte.

Para o recurso de metátese, foram as obstruintes plosivas coronais sonora e surda (ex.: quadrado $\rightarrow$ [ $\mathrm{k}^{\mathrm{w} a}$ 'dador]; litro $\rightarrow$ ['litur]), e fricativa labial sonora (ex.: livre $\rightarrow$ ['liver]) os contextos que favoreceram o emprego do recurso.

Os resultados apontam para uma possível dificuldade em relação ao traço [coronal], podendo-se considerar que haja interferência do Princípio do Contorno Obrigatório, o qual busca evitar uma sequência de traços adjacentes idênticos. Assim, ao utilizar o recurso de metátese, a criança evita 
a sequência de traços coronal, preservando o segmento, mas alterando a posição da líquida.

Esta aparente dificuldade da criança em relação ao traço [coronal] também foi verificada em uma pesquisa anterior ${ }^{8}$. A autora observou que os recursos de metátese e epêntese ocorrem mais frequentemente em palavras cuja primeira consoante é coronal, compondo uma sequência de fonemas com o mesmo traço, que parece tornar a produção da estrutura CCV ainda mais complexa.

Outra pesquisa também fortalece os achados desta, a qual verificou que o recurso de metátese envolve basicamente segmentos líquidos em posições de aquisição tardia como o onset complexo, especialmente quando são formados por duas coronais ${ }^{37}$.

Esse fenômeno pode ser explicado se for considerado que uma criança pode perceber erroneamente uma cadeia fonética, podendo realizar várias análises fonológicas ${ }^{38}$. Portanto, em onsets complexos formados por fricativas, o ruído fricativo desses segmentos distrai o ouvinte, levando a uma grande confusão com relação à ordem linear dos segmentos e à ocorrência de metátese. Este argumento parece justificar uma maior probabilidade de ocorrência de metátese em fricativas, sendo o caso das fricativas labiais sonoras deste estudo.

Variável linguística número de sílabas

As pesquisas existentes em relação à influência do número de sílabas para a produção dos segmentos são contraditórias. Alguns estudos ${ }^{6,31}$ mencionam que palavras com menor número de sílabas são as mais propensas a serem realizadas corretamente, e palavras mais extensas promovem a não realização do fonema.

Um estudo específico sobre o onset complexo ${ }^{10}$ mencionou que palavras com maior número de sílabas favorecem o cancelamento do tepe, enquanto palavras menores apresentam uma possibilidade reduzida de perder um segmento. A autora acrescenta que as palavras polissilábicas necessitam de um gerenciamento rítmico mais acurado, o que torna a sua produção ainda mais complexa.

Em relação ao comportamento desta variável para as crianças com aquisição desviante, palavras mais extensas são mais complexas para as crianças com DFE, pois quanto maior o número de sílabas, mais difícil será para a criança com aquisição desviante armazenar e repetir a palavra ${ }^{39}$.

Em relação à estratégia simplificação para $\mathrm{C}^{1} \mathrm{~V}$, os resultados da presente pesquisa concordam com estudos prévios ${ }^{6,10}$, visto que as palavras mais extensas foram mais vulneráveis a este tipo de estratégia de reparo. Além disso, considerando que a variável foi selecionada apenas para o grupo com DFE, os resultados também vão ao encontro do estudo sobre memória fononógica ${ }^{39}$, pois palavras com menor número de sílabas foram menos sujeitas às estratégias de reparo.

Diferentemente, para os recursos alteração do traço da líquida e da obstruinte, as palavras monossilábicas e dissilábicas apresentaram maior probabilidade de sofrer os recursos. Desta forma, estes achados diferem dos trabalhos supracitados ${ }^{6,10,39}$.

\section{Variável linguística contexto seguinte}

Para a estratégia simplificação para $C^{1} \mathrm{~V}$, a variável contexto seguinte foi relevante apenas para o grupo com DFN, e revelou o contexto seguinte preenchido por vogal coronal como favorecedor ao uso do recurso.

O Princípio do Contorno Obrigatório (OCP) faz referência à proibição de traços adjacentes idênticos em um determinado tier $^{40}$. Contudo, como o traço coronal da líquida está ligado ao ponto de $\mathrm{C}$ e o traço coronal da vogal está ligado a Ponto de $\mathrm{V}$, não há violação do OCP e há, ao contrário, uma facilidade articulatória. Desta forma, seria esperado que a estratégia simplificações para $\mathrm{C}^{1} \mathrm{~V}$ fosse desfavorecida na presença de contexto seguinte preenchido por vogal coronal, dado que não foi observado no presente estudo.

Em relação à estratégia alteração do traço da obstruinte, o contexto seguinte vogal dorsal apresentou maior probabilidade de ocorrência do recurso. Este resultado não seria esperado, visto que, no caso de a vogal da sílaba CCV ser dorsal, não importando a qualidade da líquida precedente, é formado um ambiente propício à produção correta do onset complexo ${ }^{8}$.

\section{Variável linguística tipo de líquida do onset complexo}

No que concerne à segunda consoante do onset complexo, variável selecionada como relevante apenas para a estratégia simplificações para $\mathrm{C}^{1} \mathrm{~V}$ no grupo com DFE, verificou-se que a líquida não lateral apresentou o maior peso relativo; no entanto, o valor ainda é considerado neutro para a aplicação da estratégia simplificação para $\mathrm{C}^{1} \mathrm{~V}$. Em contrapartida, a líquida lateral foi considerada desfavorecedora ao uso da estratégia.

A estratégia de simplificação para $C^{1} V$ é empregada de forma produtiva em ambas as qualidades de líquida, lateral e não lateral, não havendo diferenças de produções corretas entre elas ${ }^{8}$. Remetendo esses resultados para o presente estudo, pode-se observar que em relação ao uso das estratégias de reparo, o tipo de líquida exerceu um papel relevante para a criança, diferentemente dos 
achados do estudo sobre o onset complexo ${ }^{8}$, onde a qualidade da líquida não foi relevante.

Uma explicação possível seria assumir que a líquida não lateral é um segmento marcado, com propriedades mais complexas, e, portanto, de aquisição mais tardia, fato que a torna mais vulnerável às simplificações para $C^{1} \mathrm{~V}$ que a líquida lateral. Além disso, considerando que a líquida lateral é adquirida em onset simples e na coda anteriormente à líquida não lateral, pode-se pressupor que a sequência de obstruinte e líquida lateral seja menos vulnerável às estratégias de reparo e, portanto, desfavorecedora ao uso da estratégia simplificação para $\mathrm{C}^{1} \mathrm{~V}$.

\section{CONCLUSÃO}

O estudo realizado evidenciou ser a estratégia de reparo simplificação para $\mathrm{C}^{1} \mathrm{~V}$ a mais adotada por crianças com aquisição normal e desviante. Embora não tão frequentes, os recursos de alteração do traço da obstruinte, alteração do traço da líquida, metátese e epêntese também foram empregados de forma significante, e são importantes, pois indicam o modo como as crianças lidam com a estrutura complexa CCV.

A análise detalhada do uso destas estratégias de reparo revelou que há mais semelhanças do que diferenças em relação aos recursos que as crianças com DFN e DFE lançam mão no percurso de aquisição do onset complexo, quer seja em relação ao tipo de estratégias adotado ou à frequência, quer seja em relação às variantes relevantes ao seu uso.

Quanto aos fatores intervenientes, pôde-se concluir que as variáveis extralinguísticas sexo e idade, e as variáveis linguísticas tipo de obstruinte do onset complexo, tipo de líquida do onset complexo, contexto seguinte e número de sílabas influenciaram de alguma forma as estratégias de reparo analisadas. Certas variáveis atuaram de modo significante e permitiram que fossem tecidas generalizações, visto que operaram de forma semeIhante ou complementar. Sumarizando os resultados deste estudo, as conclusões encontradas foram:

- a variável sexo, quando selecionada como estatisticamente significante, mostrou ser o sexo masculino o grande favorecedor ao uso das estratégias analisadas, tanto para o grupo normal como para o grupo desviante;

- a variável idade evidenciou que, de modo geral, as estratégias de reparo empregadas no onset complexo são utilizadas de forma aleatória, por crianças de todas as faixas etárias;

- a variável tipo de obstruinte do onset complexo foi selecionada como relevante para todas as estratégias analisadas, tanto na aquisição normal como na aquisição desviante; podem-se observar comportamentos diferenciados da variável conforme o grupo analisado, DFN ou DFE, e também, de acordo com a estratégia adotada. De forma geral, as obstruintes plosivas e surdas foram mais favorecedoras às estratégias, em relação as obstruintes fricativas e sonoras; quanto ao Ponto de $\mathrm{C}$, os segmentos labiais e dorsais foram mais favorecedores;

- a variável número de sílabas foi importante apenas para as crianças com DFE, atuando de forma divergente, visto que para o recurso alteração do traço da líquida as palavras com menor extensão foram as favorecedoras, e para a estratégia simplificação para $\mathrm{C}^{1} \mathrm{~V}$ houve maior probabilidade de ocorrência da estratégia em palavras com maior extensão;

- a variável contexto seguinte foi relevante somente para as crianças com desenvolvimento fonológico normal; no entanto, não houve um padrão de comportamento em relação às estratégias analisadas, havendo maior probabilidade de ocorrer simplificação para $\mathrm{C}^{1} \mathrm{~V}$ na presença de contexto seguinte formado por vogal coronal, e maior probabilidade de ocorrer alteração do traço da obstruinte em contextos formados por vogal dorsal.

Além disso, as crianças com desenvolvimento fonológico normal lançaram mão mais frequentemente do recurso simplificação para $\mathrm{C}^{1} \mathrm{~V}$, sendo bem restrita a ocorrência de outras estratégias. Para os sujeitos com aquisição desviante, verificou-se que apesar de a estratégia simplificação para $C^{1} V$ ser a de maior ocorrência, as crianças também empregaram de forma produtiva os outros recursos observados, como alteração do traço da líquida e da obstruinte. 


\begin{abstract}
Purpose: to describe and to analyze the repair strategies used by children with normal phonological acquisition and phonological disorder in the complex onset syllable constituent, in order to examine the similarities as well as the differences in the studied groups. Method: the analysis included speech data from 60 children with normal acquisition, 30 male and 30 female, in addition to 84 children with deviation, 53 male and 31 female. The age in the normal group was between 1:0 and 4:0, whereas in the deviation group it was between 3:0 and 11:0. The variables age, gender, preceding and following context, obstruent of complex onset, kind of liquid of complex onset, tonicity, number of syllables and position in the word were considered. Then, the data were submitted to statistical analysis in the VARBRUL program. Results: the children with normal acquisition and deviation used the following resources, respectively: simplification strategies for $\mathrm{C}^{1} \mathrm{~V}(93 \% ; 77 \%)$, alteration of the obstruent feature $(5 \% ; 17 \%)$, alteration of the liquid feature $(1 \% ; 5 \%)$, epenthesis $(1 \% ; 0 \%)$ and metathesis $(0 \% ; 1 \%)$. The variables tonicity, preceding context and position in the word were not relevant to the study. Conclusion: the most frequent resource was the simplification for $\mathrm{C}^{1} \mathrm{~V}$ in both groups, but other strategies were applied, mainly in the deviation group. Considering the influence of the variables, it was possible to conclude that the variables: age, gender, kind of obstruent, kind of liquid of complex onset, following context and number of syllables influenced the studied resources.
\end{abstract}

KEYWORDS: Speech; Language Development; Child Language; Speech Disorders

\section{REFERÊNCIAS}

1. Lamprecht RR, organizador. Aquisição fonológica do Português. Perfil de desenvolvimento e subsídios para terapia. Porto Alegre: Artmed; 2004.

2. Ghisleni MRL, Keske-Soares M, Mezzomo CL. O uso das estratégias de reparo, considerando a gravidade do desvio fonológico evolutivo. Rev. CEFAC. 2010; 12 (5): 766-71.

3. Othero G. Processos fonológicos na aquisição da linguagem pela criança. [periódico online] Rev Virtual Estudos Linguag. 2005; (5): 1-13. [acesso em 15 de abril de 2009]. Disponível em: URL: http:// www.revel.inf.br.

4. Dias RF, Mota HB, Mezzomo CL. A consciência fonológica e a consciência do próprio desvio de fala nas diferentes gravidades do desvio fonológico. Rev. CEFAC. 2009 Out-Dez; 11(4):561-70.

5. Miranda ARM. A aquisição das líquidas não laterais no português do Brasil. Letras de Hoje. 1998; 33 (2): 123-31.

6. Mezzomo CL. Aquisição da coda no português brasileiro: uma análise via teoria de Princípios e Parâmetros. [tese]. Porto Alegre (RS): Pontifícia Universidade Católica do Rio Grande do Sul; 2004.

7. Vidor D. Aquisição das líquidas não laterais por crianças com desvios fonológicos evolutivos: descrição, análise, e comparação com o desenvolvimento normal. Letras de Hoje. 2001; 36(3):715-20.
8. Giacchini V, Mota HB, Mezzomo CL. Diferentes modelos de terapia fonoaudiológica nos casos de simplificação do onset complexo com alongamento compensatório. Rev. CEFAC. 2011; 13(1):57-64.

9. Gálea DES. Percurso da aquisição dos encontros consonantais, fonemas e estruturas silábicas em crianças de 2:1 a 3:0 anos de idade. Rev Soc Bras Fonoaudiol. 2009; 14(1):151.

10. Ribas L. Onset complexo: características da aquisição. Letras de Hoje. 2003; 38(2):23-31.

11. Ribas L. Onset complexo nos desvios fonológicos: descrição, implicações para a teoria, contribuições para terapia [tese]. Porto Alegre (RS): Pontifícia Universidade Católica do Rio Grande do Sul; 2006.

12. Miranda I. Aquisição e variação estruturada de encontros consonantais tautossilábicos. [tese]. Belo Horizonte (MG): Universidade Federal de Minas Gerais; 2007.

13. Cavalheiro LG, Keske-Soares M. Prevalência do desvio fonológico em crianças de 4 a 6 anos de idade. Pró-Fono. 2008; 20 (supl.): 11-3.

14. Attoni TM, Albiero JK, Berticelli A, Keske-Soares M, Mota HB. Onset complexo pré e pós-tratamento de desvio fonológico em três modelos de terapia fonológica Rev Soc Bras Fonoaudiol. 2010;15(3):395-400.

15. Lamprecht $R R$. Diferenças no ranqueamento de restrições com origem de diferenças na aquisição fonológica. Letras de Hoje. 1999; 34(3):65-82.

16. Keske-Soares M, Lamprecht RR, Pagliarin KC, Ghisleni MRL. Aquisição não-linear durante 
o processo terapêutico. Letras de Hoje. 2008; 43(3):22-6.

17. Grunwell $P$. Os desvios fonológicos evolutivos numa perspectiva linguística. In: Yavas $M$, organizador. Desvios fonológicos em crianças. Porto Alegre: Mercado Aberto; 1990; 51-82.

18. Wertzner HF, Pagan LO, Galea DES, Papp ACCS. Características fonológicas de crianças com transtorno fonológico com e sem histórico de otite média. Revista da Sociedade Brasileira de Fonoaudiologia. 2007;12(1):41-7

19. Wertzner HF, Amaro L, Galea DE. Phonological performance measured by speech severity indices compared with correlated factors. São Paulo Med J. 2007; 125(6): 309-14.

20. Schriberg LD. Five subtypes of developmental phonological disorders. Clinics in Communication Disorders. 1994; 4: 38-53.

21. Schriberg LD, Lewis BA, McSweeny JL, Wilson DL. The speech disorders classification system (SDCS): Extensions and lifespan reference data. Journal of Speech, Language, and Hearing Research. 1997; 40: 723-40.

22. Yavas M, Hernandorena C, Lamprecht R. Avaliação fonológica da criança. Porto Alegre: Artes Médicas; 1991.

23. Cedergren HJ, Sankoff D. Variable rules: performance as a statistical reflexion of competence. Language. 1974; 50(2):332-55.

24. Scherre M. Introdução ao Pacote VARBRUL para microcomputadores. Rio de Janeiro: UFRJ; 1993.

25. Ferrante C, Borsel J, Pereira M. Análise dos processos fonológicos com desenvolvimento fonológico normal. Rev Soc Bras Fonoaudiol. 2009; 14(1):36-40.

26. Patah LK, Takiuchi N. Prevalência das alterações fonológicas e uso dos processos fonológicos em escolares aos 7 anos. Rev. CEFAC. 2008; 10(2):158-67.

http://dx.doi.org/10.1590/S1516-18462012005000084

Recebido em: 24/08/2011

Aceito em: 22/12/2011

Endereço para correspondência:

Simone Weide Luiz

Rua Barão do Triunfo, 1710/202

Santa Maria - RS

CEP: 97015-070

E-mail: simonewluiz@ hotmail.com
27. Mann W, Marshall CR, Mason K \& Morgan

G. The acquisition of sign language: The impact of phonetic complexity on phonology. Language Learning and Development. 2010; 6: 60-86.

28. Keske-Soares M, Mota HB, Pagliarin KC. Estudo sobre os ambientes favoráveis à produção da líquida não-lateral /r/ no tratamento do desvio fonológico. Rev Soc Bras Fonoaudiol. 2007; 12(1):48-54.

29. Mota H. Aquisição segmental do Português: um modelo implicacional de complexidade de traços. Letras de Hoje. 1997; 32(4):23-47.

30. Mezzomo C, Baesso J, Athayde M, Dias R, Giacchini V. O papel do contexto fonológico no desenvolvimento da fala: implicações para a terapia dos desvios fonológicos evolutivos. Letras de Hoje. 2008; 43 (3): 15-21.

31. Marshall CR. van der Lely HKJ. Effects of word position and stress on onset cluster production: Evidence from typical development, SLI and dyslexia. Language. 2009; 85: 39-57.

32. Mezzomo C, Mota H, Dias R, Giacchini V. O uso da estratégia de alongamento compensatório em crianças com desenvolvimento fonológico normal e desviante. Letras de Hoje. 2008; 43 (3): 35-41.

33. Ferrante C, Borsel J, Pereira M. Aquisição fonológica de crianças de classe sócio econômica alta. Rev CEFAC. 2008; 10 (4): 452-60.

34. Vito, R, Cardoso-Martins C. Desenvolvimento fonológico de crianças pré-escolares da Região Noroeste de Belo Horizonte. Psicologia em Revista. 2007; 13 (2): 383-98.

35. Mota $\mathrm{H}$, Athayde M, Mezzomo CL. O acesso ao léxico em crianças com desenvolvimento fonológico normal e desviante. Letras de Hoje. 2008; 43(3): 54-60.

36. Magalhães J. Produção de oclusivas mais líquida não lateral e consciência fonológica na fala de crianças em aquisição de linguagem: análise pela geometria de traços [dissertação]. Uberlândia (MG): Universidade Federal de Uberlândia; 2000.

37. Zitske BC. Um levantamento de metáteses na fala de crianças em fase de aquisição de linguagem. Letras de Hoje. 2001; 36(3):219-22.

38. Blevins J, Garret A. The evolution of metathesis. In: Hayes B, Kirschner R, Steriade D, organizadores. Phonetically based phonology. Cambridge: Cambridge University Press; 2004; 117- 56.

39. Linassi L, Keske-Soares M, Mota H. Habilidades de memória de trabalho e o grau de severidade do desvio fonológico. Pró-Fono. 2005; 17(3):383-92.

40. Clements GN, Hume EV. The internal organization of speech sounds. In: Goldsmith J, organizador. The handbook of phonological theory. London: Basil Blackwell; 1995. 